\title{
Nitrobutadienes from $\beta$-nitrothiophenes: valuable building-blocks in the overall ring-opening / ring-closure protocol to homo- or hetero-cycles
}

\author{
Lara Bianchi, ${ }^{a}$ Carlo Dell'Erba, ${ }^{a}$ Massimo Maccagno, , Stefano Morganti, \\ Giovanni Petrillo, ${ }^{*}$ a Egon Rizzato, ${ }^{\text {b }}$ Fernando Sancassan, ${ }^{\text {a Elda Severi, }}{ }^{\text {a }}$ \\ Domenico Spinelli, ${ }^{b}$ and Cinzia Tavani ${ }^{a}$ \\ ${ }^{a}$ Dipartimento di Chimica e Chimica Industriale, Università di Genova, Via Dodecaneso 31, \\ I-16146 Genova, Italy \\ ${ }^{b}$ Dipartimento di Chimica Organica “A. Mangini”, Università di Bologna, Via San Giacomo 11, \\ I-40126 Bologna, Italy \\ E-mail:petrillo@chimica.unige.it
}

\begin{abstract}
$\beta$-Nitrothiophenes undergo a facile ring-opening process when treated with secondary amines in ethanol (in the presence of $\mathrm{AgNO}_{3}$, but for 3,4-dinitrothiophene), providing interesting polyfunctionalized linear nitro- or dinitro-butadienic building-blocks, which can be further manipulated and eventually exploited for the preparation of targets of various nature. In particular, a great variety of homo- and hetero-cyclic compounds may be obtained and some representative results in this field, relevant to synthetic, mechanistic and stereochemical aspects, are reported herein.
\end{abstract}

Keywords: Nitrothiophenes, ring-opening, ring-closure, heterocycles, nitrobutadienes, isoxazoles

\section{Contents}

1. Introduction

2. The ring-opening of nitrothiophenes

2.1. Ring-opening of 3,4-dinitrothiophene

2.2. Ring-opening of 2-nitrothiophene

2.3. Ring-opening of $\beta$-nitrothiophenes other than 3,4-dinitrothiophene

3. Exploitation of the synthetic potentialities of nitrobutadienic building-blocks

3.1. Synthetic exploitation of 1,4-bis(dialkylamino)-2,3-dinitro-1,3-butadienes

3.1.1. Monooximes and dioximes as precursors of heterocycles 
3.1.2. Monocyclopropanes, bi(cyclopropyl)s and heterocycles therefrom

3.1.3. Pyrrolines, pyrrolidines and pyrroles

3.1.4. Ethynylpyrroles

3.2. Synthetic exploitation of nitrobutadienes from $\beta$-nitrothiophenes other than 3,4dinitrothiophene

3.2.1. Thiochromans from 3-nitrobenzo[b]thiophene

3.2.2. Homo- and hetero-cycles from $\beta$-nitrothiophenes. A dichotomic thermal vs. baseinduced cyclization

3.2.3. Isoxazoles from 2-methylthio-4-nitrothiophene

\section{Introduction}

The ring-opening of nitrothiophenes and the synthetic exploitation of the ensuing nitro- or dinitro-butadienes as building-blocks for the synthesis of both linear and homo- or hetero-cyclic targets has represented for over 30 years one of the most rewarding research subjects of our group. Actually, our involvement in such versatile chemistry started rather casually some years ago, in the course of a more comprehensive study on the behaviour of some electron-deficient thiophene derivatives towards nucleophiles of different nature. Thus, in 1969 3,4dinitrothiophene $(3,4-\mathrm{DNT}, \mathbf{1})$ manifested a peculiar non-benzenoid character (Scheme 1) as cine-substitution was observed in its reaction with sulfur nucleophiles, ${ }^{1}$ while ring-opening was the unexpected result of the reactions with nitrogen nucleophiles such as primary or secondary amines. ${ }^{2}$ Another aspect of the non-benzenoid behaviour of 3,4-DNT is a more recent finding, concerning the reactivity towards carbon nucleophiles like Grignard reagents, leading to the interesting aminonitroderivatives $\mathbf{4}$ as the result of a rather complex process, characterized by a pivotal Claisen-like rearrangement. ${ }^{3}$

The ring-opening of 3,4-DNT put in our hands a nice butadienic polyfunctionalized fragment, whose wide reactivity has been deeply studied since, developing convenient synthetic accesses to variously substituted linear and cyclic compounds. ${ }^{4}$ The extension of the ringopening reaction to 2-nitrothiophene $(2-\mathrm{NT}),{ }^{5} 3$-nitrothiophene (3-NT) and substituted $\beta$ nitrothiophenes ( $\beta$-NT's $)^{6}$ other than $3,4-\mathrm{DNT}$ are more recent results in the field; in all these cases the process is substantially different from that operating for 3,4-DNT, but nonetheless variously functionalized butadienic fragments were obtained, which could be elaborated to give in turn acyclic, homocyclic and heterocyclic derivatives. ${ }^{7}$ 


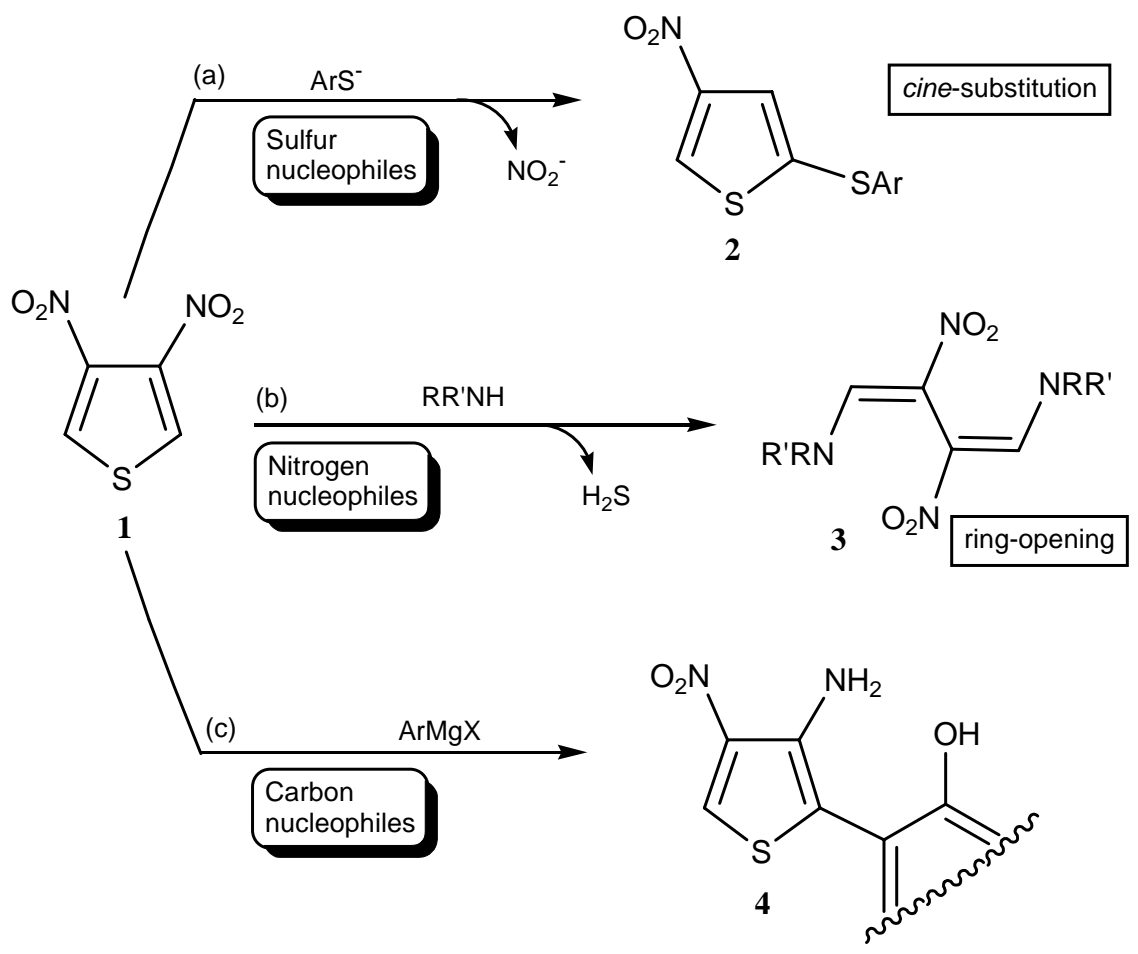

Scheme 1. Non-benzenoid reactivity of 3,4-dinitrothiophene.

\section{The ring-opening of nitrothiophenes}

\subsection{Ring-opening of 3,4-dinitrothiophene}

When an excess of a secondary amine is added to a solution of 3,4-DNT in ethanol, after some time crystals can be collected of the ring-opening product 1,4-bis(dialkylamino)-2,3-dinitro-1,3butadiene (5), where sulfur has been extruded as hydrogen sulfide and two dialkylamino groups have been attached to $\mathrm{C}(1)$ and $\mathrm{C}(4)$ (Scheme 2). The conjugated nitroenamino functionalities of compounds 5 make them interesting intermediates because of their wide synthetic potentialities: by reaction with carbon nucleophiles, they allow, e.g., elongation of the carbon skeleton before further elaboration (see paragraph 3.1 below).

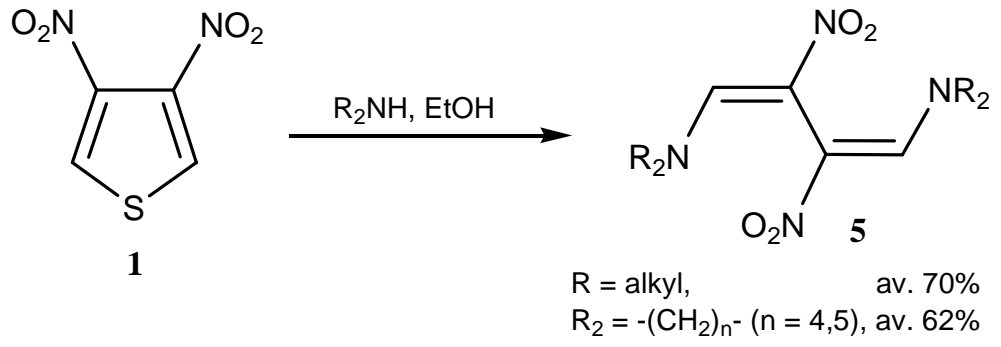

Scheme 2. Ring-opening of 3,4-dinitrothiophene. 


\subsection{Ring-opening of 2-nitrothiophene}

In $1974^{5}$ the reaction with secondary amines was extended to 2-nitrothiophene (2-NT, 6), finding that in this case, at variance with what observed with 3,4-DNT, sulfur is not extruded. The reaction (Scheme 3) proceeds through the breakage of just one carbon-sulfur bond, generating, as the final product, a nitrobutadienedisulfide (7); in the presence of silver nitrate, the butadienethiole intermediate can be isolated as the silver thiolate $\mathbf{8}$ which can be reacted with MeI to give the corresponding methylthio derivative $\mathbf{9}$. It was also verified that the silver thiolate can be conveniently trapped with MeI with no need for isolation.

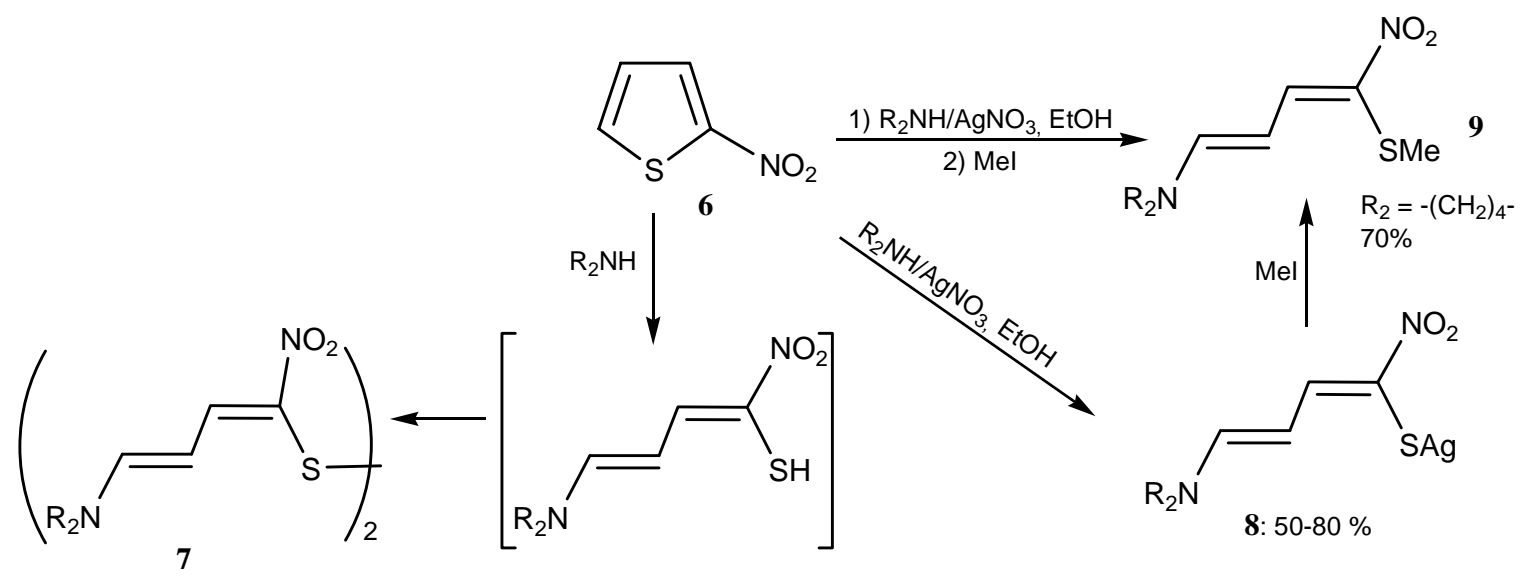

Scheme 3. Ring-opening of 2-nitrothiophene.

\subsection{Ring-opening of $\beta$-nitrothiophenes other than 3,4-dinitrothiophene}

In 1997 it was reported by others that the experimental conditions employed by us for the opening of 2-nitrothiophene could also be applied, although with moderate success, to 3nitrothiophene; ${ }^{8}$ later, we optimized the conditions for the opening of 3-NT, successfully extending the process to a number of variously 2- and/or 3-substituted 4-nitrothiophenes (Scheme 4). ${ }^{6}$ In a typical procedure, the $\beta$-NT and silver nitrate are dissolved under argon in absolute ethanol, and pyrrolidine is added; after 30' of sonication, the reaction mixture is left overnight at room temperature in the dark. Usually MeI is added with no need to isolate the silver salt, leading directly to the recovery of the methylthioderivative $\mathbf{1 1 .}$

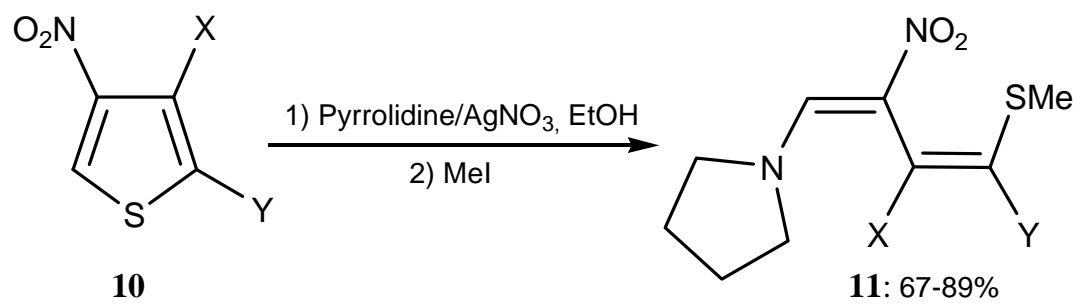

Scheme 4. Ring-opening of $\beta$-nitrothiophenes other than 3,4-DNT. 


\section{Exploitation of the synthetic potentialities of nitrobutadienic building- blocks}

As already pointed out, the ring-opening of nitrothiophenes produces appealing building-blocks encompassing a number of versatile conjugated functionalities. A systematic exploitation of their synthetic possibilities began about fifteen years ago for the dinitrobutadienes $\mathbf{5}$ deriving from 3,4-DNT and only some ten years later for mononitrobutadienes deriving from 3-NT and other $\beta$-NT's (11). As far as building-blocks from 2-NT (9) are concerned, we are still at a very initial stage, but they seem to be as much promising as the others. ${ }^{9}$

We have targeted our investigations essentially to three main goals, namely:

- $\quad$ synthesis of open-chain polyfunctionalized molecules;

- $\quad$ synthesis of homo- and hetero-cyclic derivatives;

- investigation of possible pharmacological applications of some of the compounds obtained.

Actually, the synthesis of cyclic derivatives, which, starting from a properly substituted thiophene ring, can be envisaged as an overall ring-opening / ring-closure process (Scheme 5), has so far proved to be a very fertile field and a number of appealing applications have been optimized in the last few years.

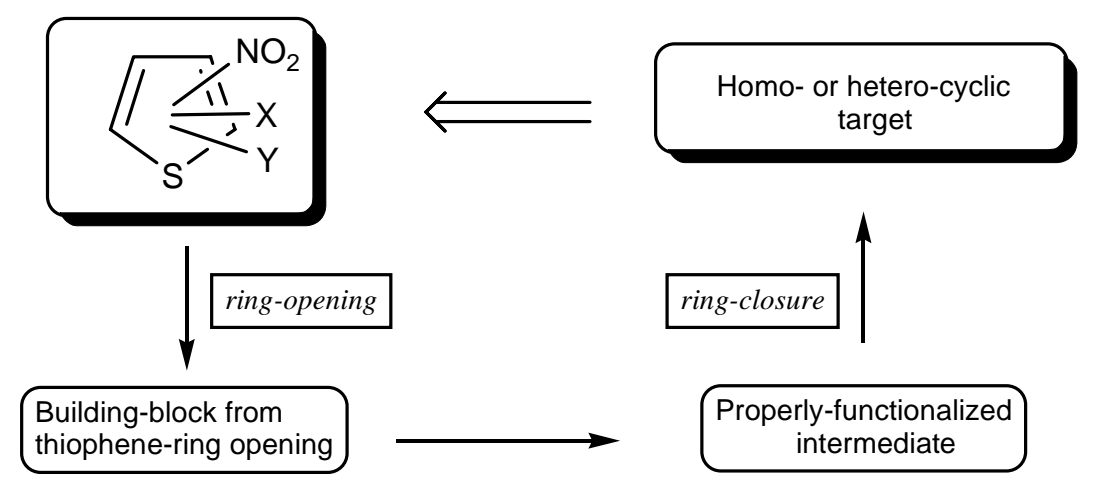

Scheme 5. The ring-opening / ring-closure synthetic protocol.

\subsection{Synthetic exploitation of 1,4-bis(dialkylamino)-2,3-dinitro-1,3-butadienes}

The synthetic potentialities offered by the ring-opening products 5 of 3,4-DNT have been extensively examined by us since 1990, and an overview of the main results so far obtained within the overall ring-opening / ring-closure protocol of Scheme 5 is depicted in Schemes 6A6C. The initial step in the syntheses shown is always represented by the practically quantitative replacement of the two diethylamino groups by reaction with an organometallic compound, namely an aryl (Scheme 6A), ${ }^{10}$ vinyl (Scheme 6B) ${ }^{11}$ or benzyl Grignard reagent (Scheme 6C). ${ }^{12}$ In the latter two cases, nitrosubstituted carbocyclic compounds have been eventually obtained, as the result of interesting processes encompassing electrocyclization steps followed or not by 
aromatization. Hereinafter in the paragraph the achievements reported in Scheme 6A, relevant to the synthesis of heterocycles, will be discussed in more details.

It should anyway be noticed that, as a common feature to Scheme 6A-6C, the diethylamino group of $\mathbf{5 a}$ has proved to be the best compromise, from a practical point of view, between the yield of the ring-opening of 3,4-DNT and that relevant to the replacement of the aminogroup itself.

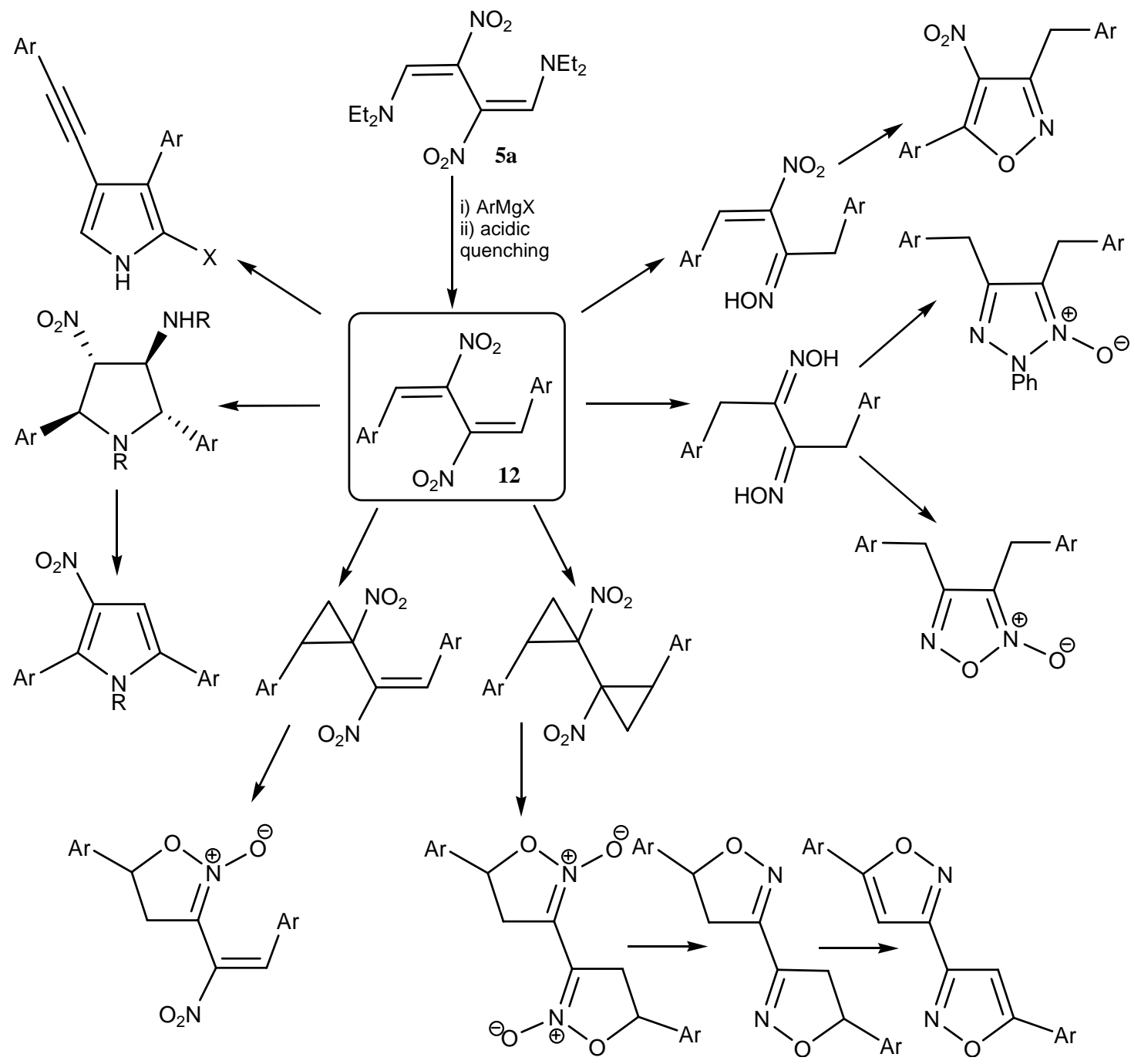

Scheme 6A. A panorama of the versatility of 1,4-diaryl-2,3-dinitro-1,3-butadienes as precursors of heterocycles.

As it clearly shows up from Schemes 6A-6C, the treatment of $\mathbf{5}$ with organometals usually leads to butadienes which possess two conjugated identical nitrovinyl moieties, although the possibility to introduce different aryl groups at the two ends of the butadienic chain has been successfully verified. ${ }^{4 b, 13}$ In the follow-up procedures, the two nitrovinyl functionalities may react independently or show cooperative/sinergic effects; quite appealingly, they can also be 
differentiated by a proper choice of reactants or of experimental conditions. Some representative examples of such possibilities are reported below.
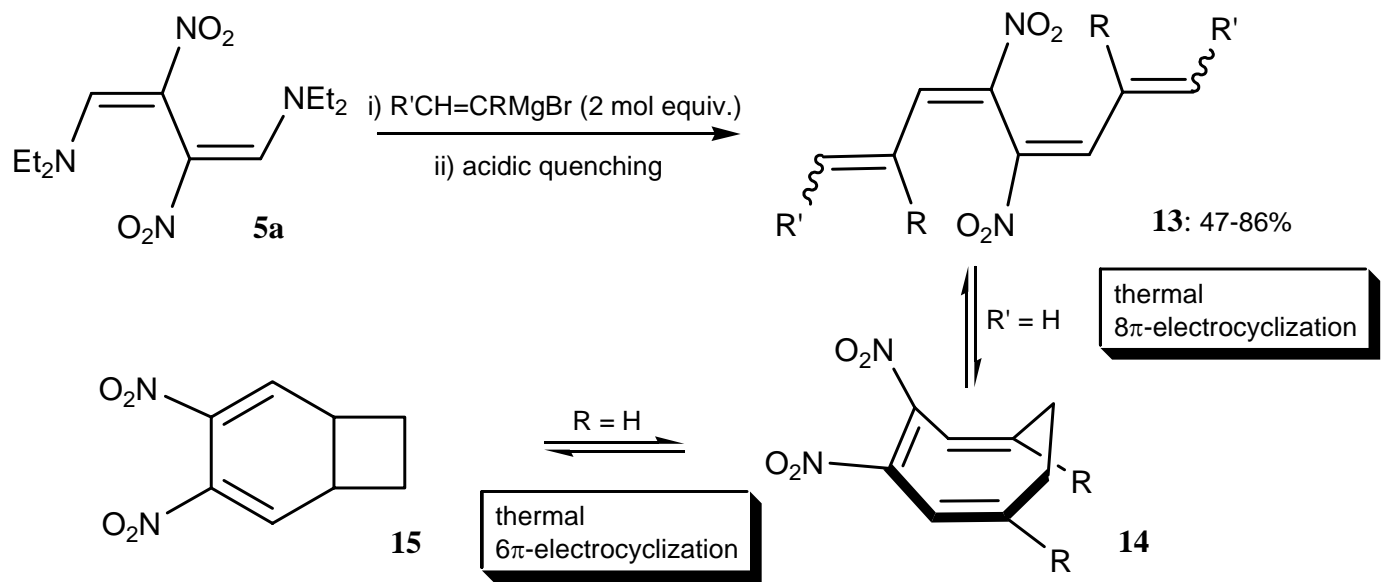

Scheme 6B. Synthetic exploitation of dinitrobutadienes from 3,4-DNT.

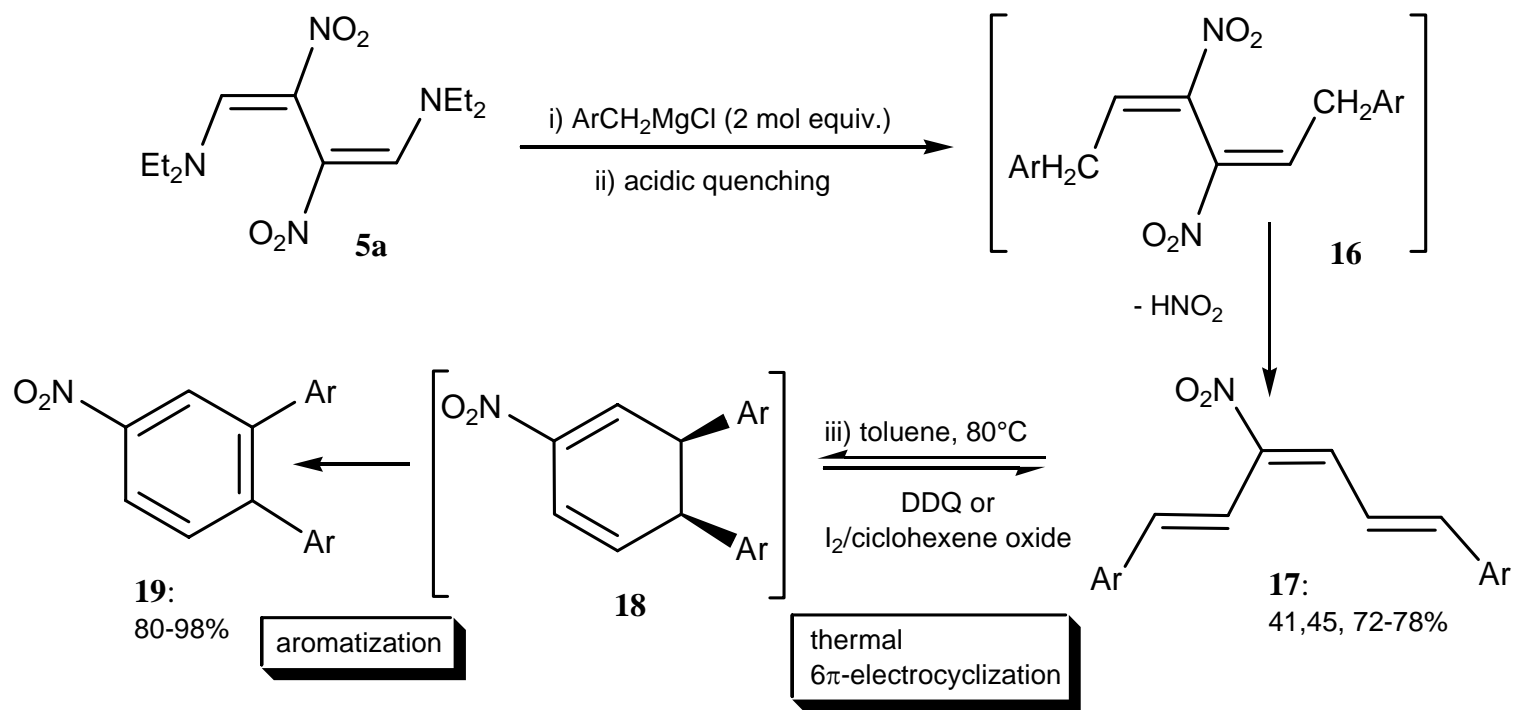

Scheme 6C. Synthetic exploitation of dinitrobutadienes from 3,4-DNT.

\subsubsection{Monooximes and dioximes as precursors of heterocycles}

The two nitrovinyl functionalities of compounds $\mathbf{1 2}$ may undergo reduction to nitrovinyloximes or to dioximes, depending on the choice of the reagent. Thus, if the reduction is performed in acidic conditions by stoichiometric stannous chloride, nitrovinyloximes $\mathbf{2 0}$ can be obtained, as precursors, through cyclization and aromatization, of the polysubstituted isoxazoles $\mathbf{2 1}$ (Scheme 7). ${ }^{14}$ 


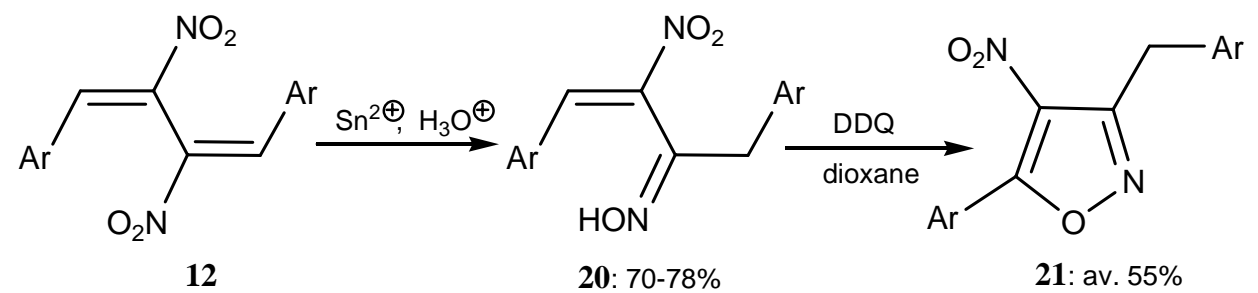

Scheme 7. From 1,4-diaryl-2,3-dinitro-1,3-butadienes to 4-nitroisoxazoles.

On the other hand, reduction with lead in DMF leads (Scheme 8) to symmetrical dioximes 22, ${ }^{10}$ which are precursors of other nitrogen heterocycles such as the $\mathrm{N}$-oxides of oxadiazoles (23) or of triazoles (26) (in the latter case through a previous differentiation of the two functionalities by hydrolysis to ketooximes $\mathbf{2 4}$, followed by transformation into the corresponding hydrazonooximes 25). ${ }^{15}$

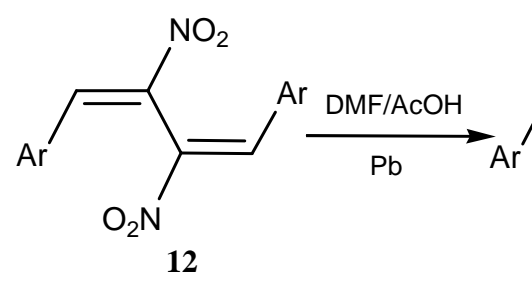

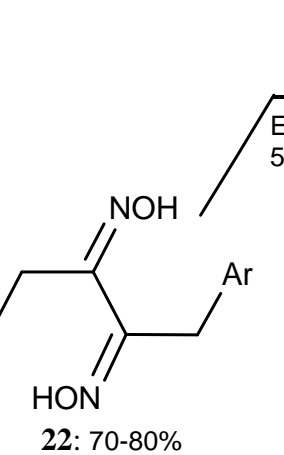

HON

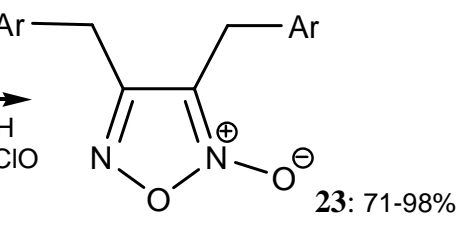

$\operatorname{Ar}$

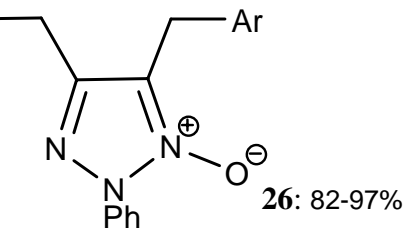

Scheme 8. From 1,4-diaryl-2,3-dinitro-1,3-butadienes to oxadiazoles and triazoles.

\subsubsection{Monocyclopropanes, bi(cyclopropyl)s and heterocycles therefrom}

A selective functionalization of the two conjugated nitrovinyl moieties of dinitrobutadienes $\mathbf{1 2}$ can also be obtained with a proper choice of experimental conditions such as the reactant to substrate ratio: for instance (Scheme 9), cyclopropanation of $\mathbf{1 2}$ with diazomethane occurs in two consecutive steps and can be preparatively stopped at the monocyclopropanation level (to give the nitrovinylcyclopropanes 27) when just one equivalent of reactant is used in controlled conditions. Bi(cyclopropyl) derivatives 28 may be prepared, on the other hand, if excess 
diazomethane is employed; compounds $\mathbf{2 8}$ may undergo a sequence of transformations, eventually leading to the symmetrical bis-isoxazoles $31 .^{16}$ The nitrocyclopropyl moiety of derivatives 27 likewise isomerizes with $\mathrm{NaI}$, but, surprisingly enough, the resulting isolable isoxazoline $\mathrm{N}$-oxides 32 further react, in the same experimental conditions, to give, through an unprecedented nitrogroup migration, the isomeric isoxazolines $33 .^{17}$

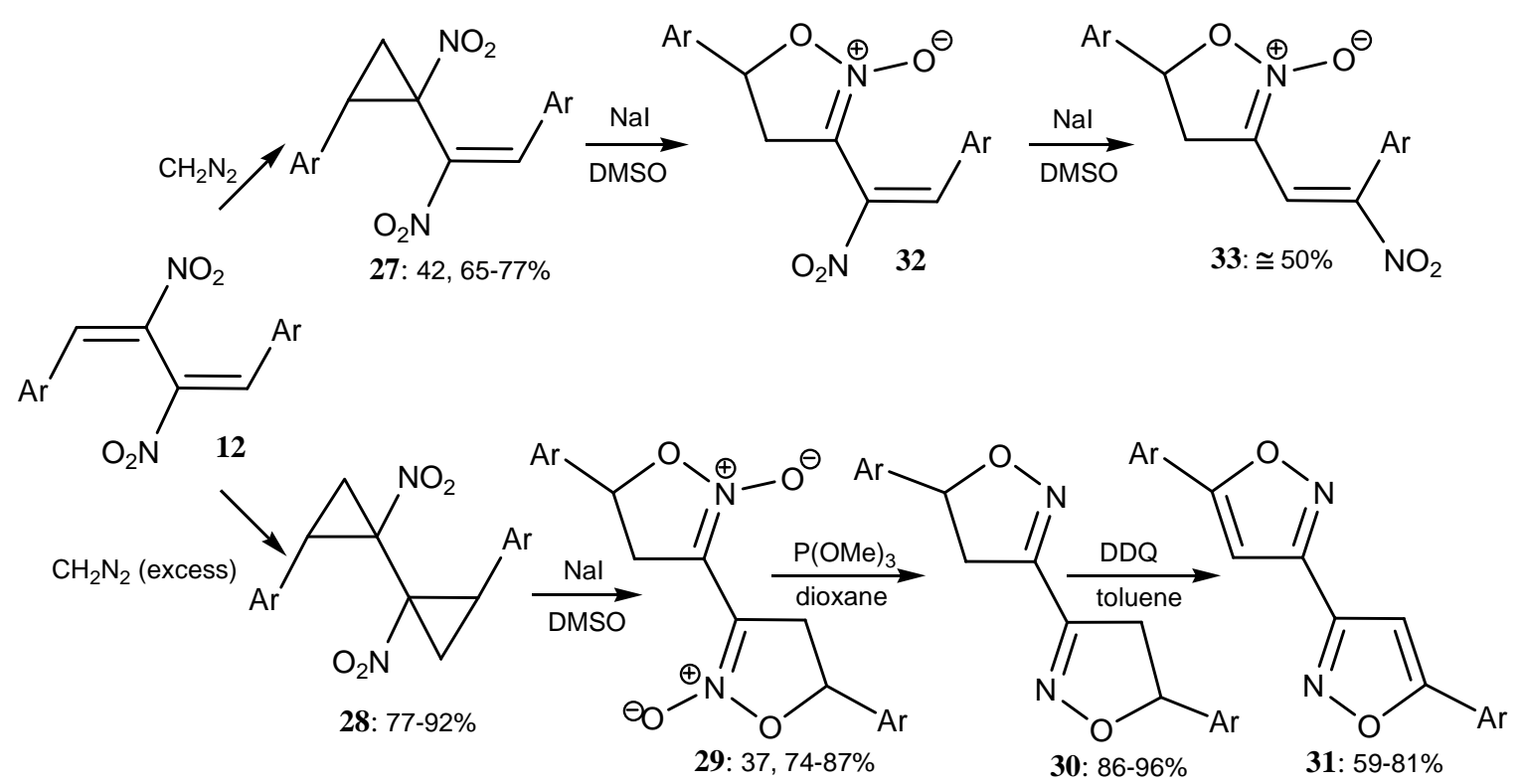

Scheme 9. From 1,4-diaryl-2,3-dinitro-1,3-butadienes to homo- and hetero-cycles.

The 12 to 27 monocyclopropanation allows the differentiation of the two originary nitrovinyl moieties. Anyway, as pointed out above, such a goal can also be reached at a previous stage, using one equivalent of a hindered Grignard reagent, such as the mesityl derivative, for a selective replacement of a single amino group (Scheme 10). If the second amino group is replaced by a different Grignard reagent, the following cyclopropanation is in turn selectively directed towards the less hindered nitrovinyl moiety. ${ }^{18}$ 


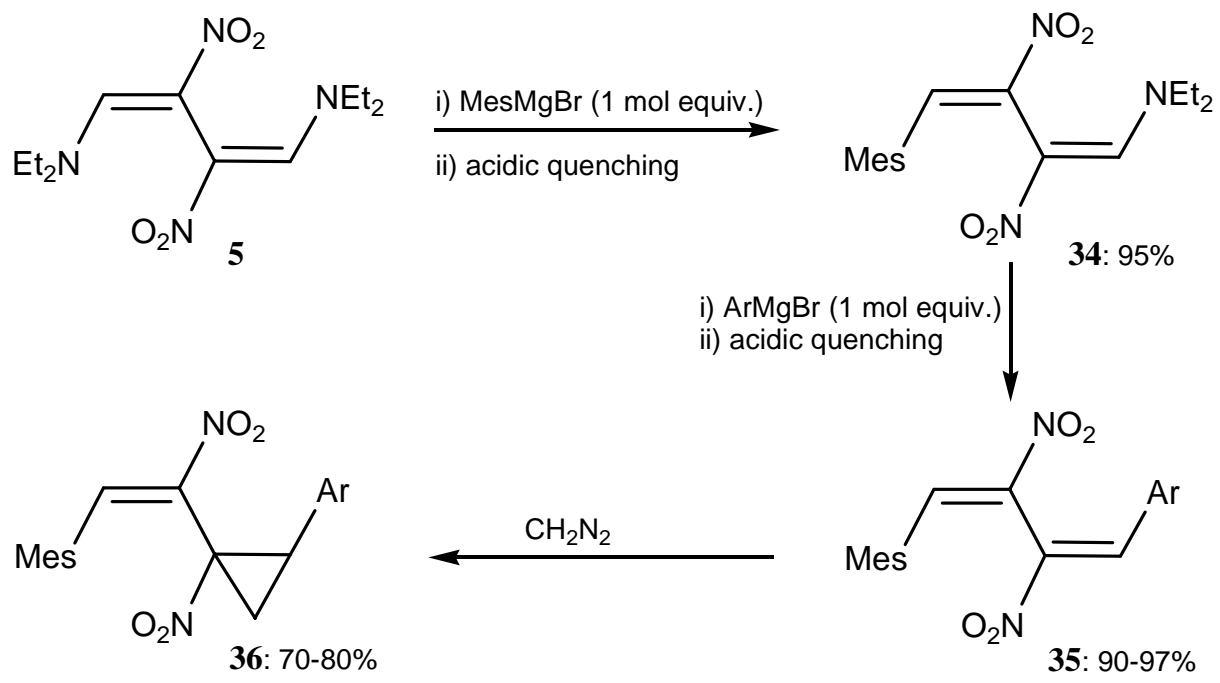

Scheme 10. Selective cyclopropanation of differently hindered nitrovinyl moieties.

\subsubsection{Pyrrolines, pyrrolidines and pyrroles}

Another convenient access to nitrogen heterocycles is represented by the reaction of compounds 12 with primary amines (Scheme 11). As a matter of fact, the reaction proceeds in very mild conditions following a multi-step pathway via a pivotal, unusually favoured, 5-endo-trig cyclization, allowing a high-yielding construction of a polysubstituted pyrrolidine ring (37) from which pyrrolines (38) and pyrroles (39) can be easily obtained by means of common procedures in fair to quantitative yields. The reactions can be applied even to differently functionalized primary amines such as allylamine, propargylamine and ethanolamine, while less nucleophilic amines (Ar-NH 2 and $\left.\mathrm{Bu}^{\mathrm{t}}-\mathrm{NH}_{2}\right)$ are unreactive. ${ }^{19}$<smiles>O=[N+]([O-])C(=C/[Al])/C(=C\[17Cl])[N+](=O)[O-]</smiles>

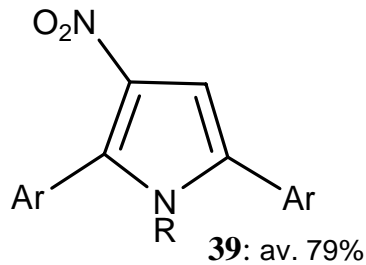

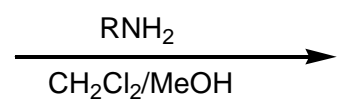

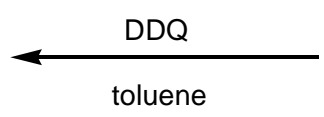

toluene

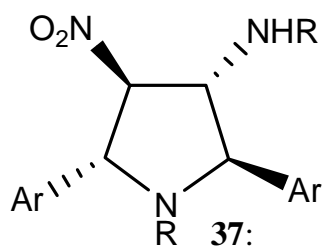

$42,45,85-98 \%$

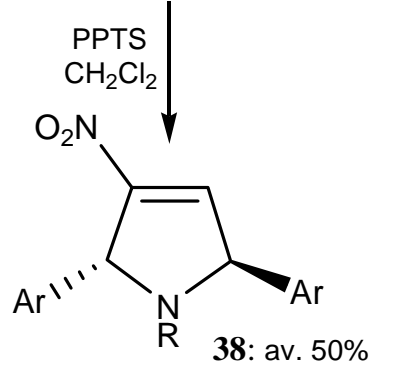

Scheme 11. 1,4-Diaryl-2,3-dinitro-1,3-butadienes as precursors of pentatomic nitrogen heterocycles. 


\subsubsection{Ethynylpyrroles}

This reaction, which leads as the final products (Scheme 12) to the 2-aryl-3-ethynylpyrroles $\mathbf{4 0 ,}$ is a clear example of cooperation between the two nitrovinyl moieties. In fact, the nucleophilic addition of an isocyanide anion on a nitroalkene is followed by an unexpected elimination of nitrous acid which brings to the formation of an ethynyl nitronate, precursor of the pyrrole ring. ${ }^{20}$
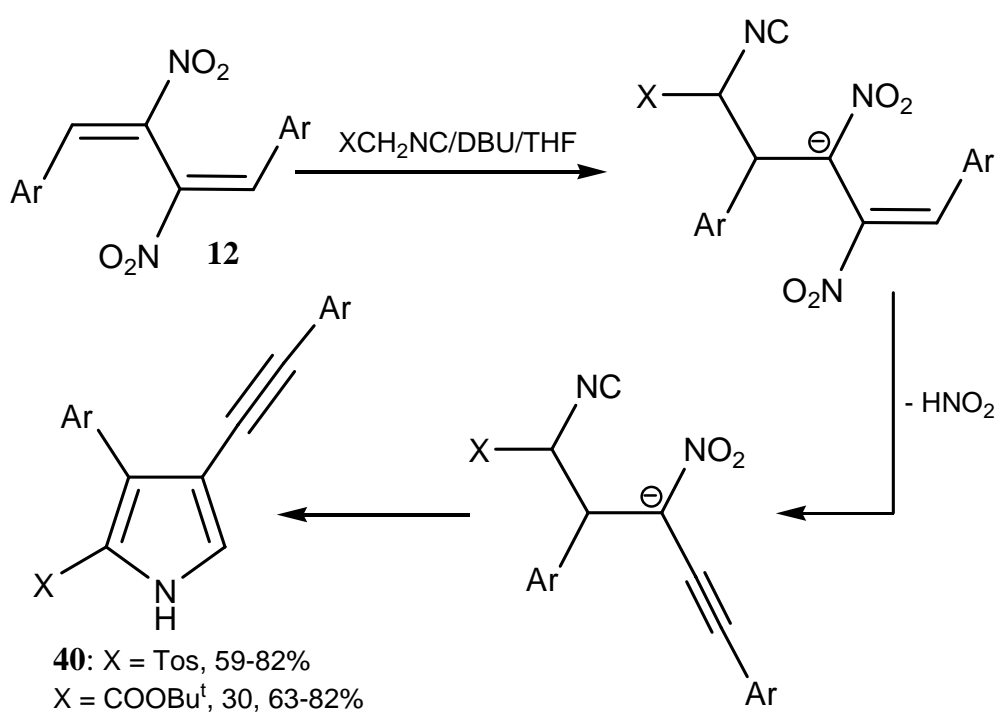

Scheme 12. Ethynylpyrroles from 1,4-diaryl-2,3-dinitro-1,3-butadienes.

\subsection{Synthetic exploitation of nitrobutadienes from $\beta$-nitrothiophenes other than 3,4 - dinitrothiophene}

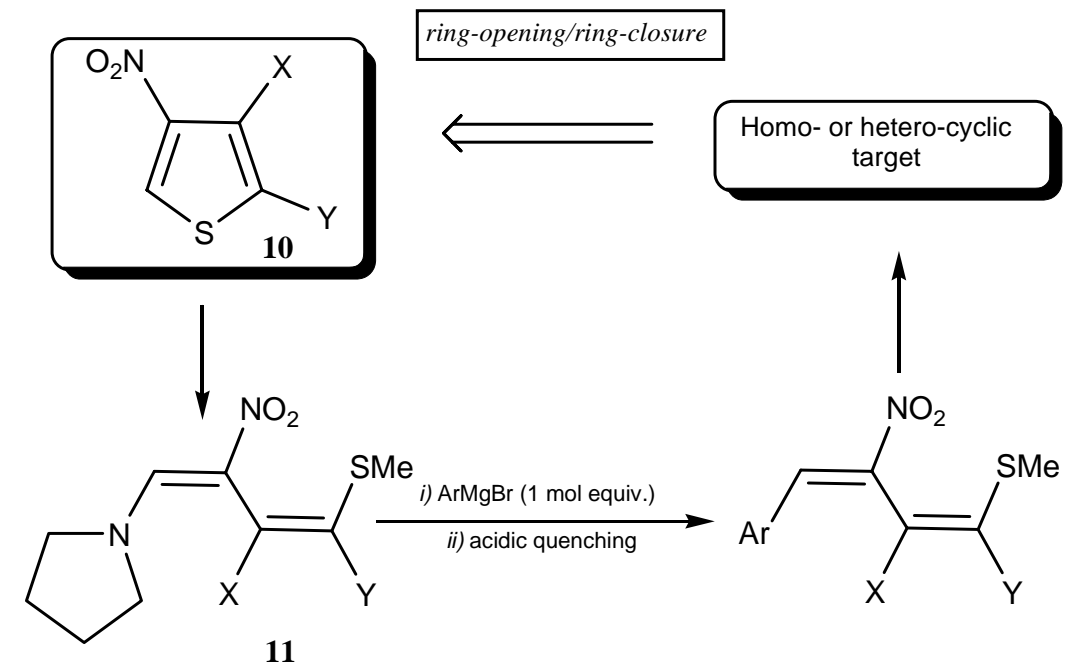

Scheme 13. The ring-opening / ring-closure synthetic protocol through open-chain nitrobutadienic derivatives from $\beta$-nitrothiophenes. 
The treatment of the 1-pyrrolidino-2-nitrobutadienes 11 (obtained from the ring-opening of $\beta$ nitrothiophenes: cf. Scheme 4) with 1 molar equivalent of an aryl Grignard reagent leads chemoselectively and almost quantitatively to the replacement of the pyrrolidino group, maintaining in the product the original E-stereochemistry of the attacked nitroenamino doublebond (Scheme 13). The polyfunctionalized building-blocks obtained in this way are in turn amenable to a number of applicative transformations. Some recent results relevant to the synthesis of homo- or hetero-cyclic compounds via the ring-opening / ring-closure procedure from $\mathbf{1 0}$ are reported in the following paragraphs.

\subsubsection{Thiochromans from 3 -nitrobenzo $[b]$ thiophene}

The condensed heterocycle 3-nitrobenzo[b]thiophene 41 undergoes, much as simple $\beta$ nitrothiophenes, ring-opening ${ }^{8 b}$ when reacted with pyrrolidine and silver nitrate (Scheme 14); after methylation of the non-isolated silver thiolate it is possible to recover in high yields a "butadiene" system in which one double bond is part of a phenyl ring.

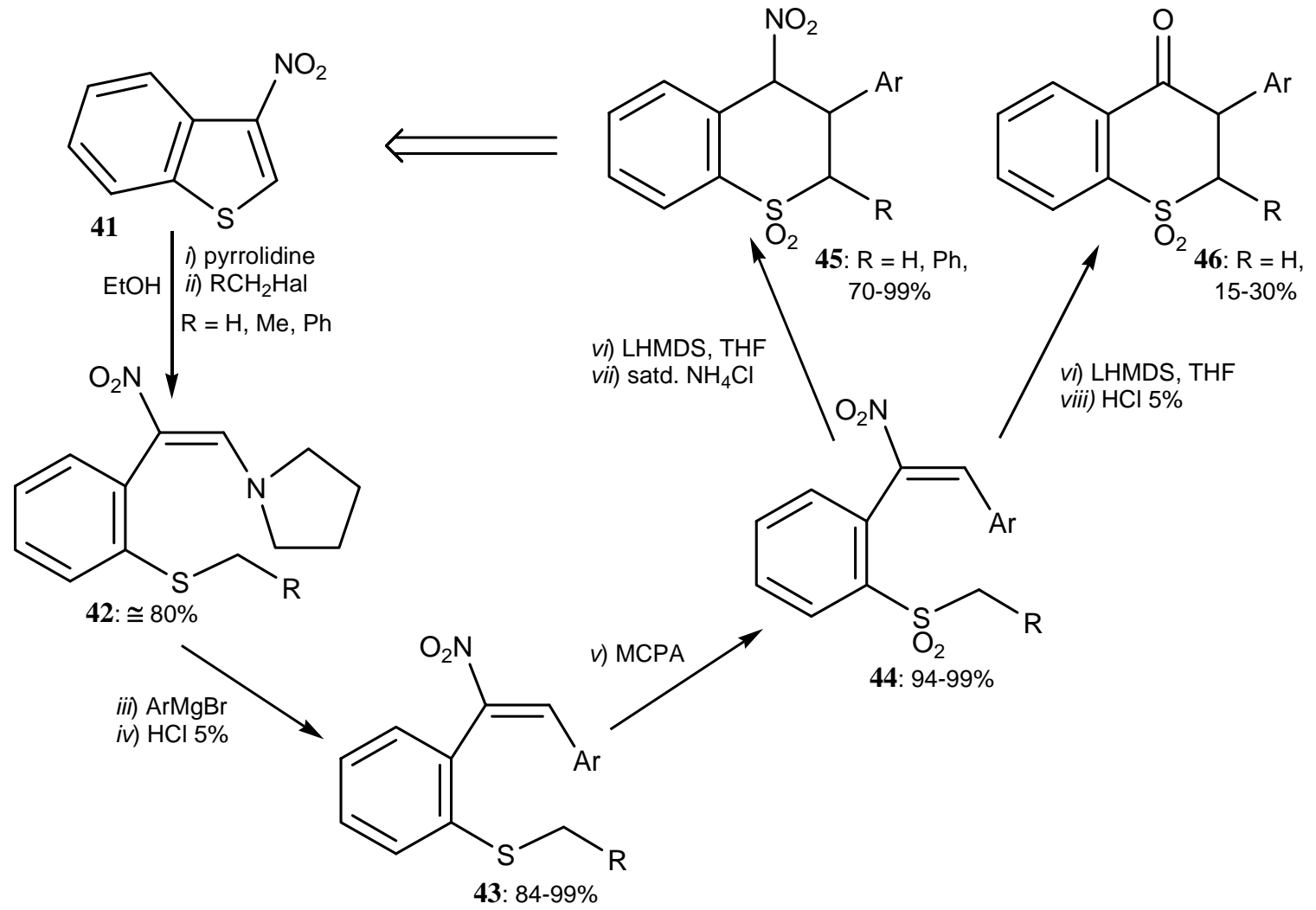

Scheme 14. Thiochroman derivatives from the ring-opening products of 3nitrobenzo[b]thiophene.

Replacement of the pyrrolidino with an aryl group by reaction with an aryl Grignard reagent followed by oxidation of the sulfur atom leads to the intermediate $\mathbf{4 4}$, endowed with a 
sulfonyl-activated methylene group: treatment with LHMDS at $0{ }^{\circ} \mathrm{C}$ followed by acidic quenching eventually brings to the thiochroman S,S-dioxides 45 (Scheme 14). ${ }^{7 \mathrm{~b}}$ The cyclization step still needs optimization for $\mathrm{R}=\mathrm{Me}$, while for $\mathrm{R}=\mathrm{H}$ or Ph yields are generally excellent. The process can be quite straightforwardly explained as an intramolecular Michael-type addition to the nitrovinyl moiety of the initially formed sulfonyl-stabilized anion; stereochemical aspects should be considered as the reaction generates two or three stereocenters, depending on the nature of $\mathrm{R}$. If $\mathrm{R}=\mathrm{H}$, two diastereomeric racemic mixtures are always isolated with low stereoselectivity, corresponding to the cis and trans configurations at the $\mathrm{C}(3)-\mathrm{C}(4)$ bond, with a slight preference for the cis-racemate. ${ }^{7 b}$ When $\mathrm{R}=\mathrm{Ph}$, the situation results more complex but promises to be more interesting from a speculative point of view, as only three of the four possible diastereoisomers are present throughout, in relative ratios which depend on the reaction temperature. The matter is presently under investigation. ${ }^{21}$ Another interesting aspect is that this intramolecular cyclization produces different derivatives depending on the nature of the acidic quenching: the nitrothiochromans $\mathbf{4 5}$ are obtained when ammonium chloride is used as the acid, while the corresponding thiochromanones $\mathbf{4 6}$ are obtained, although in less satisfactory yields, with 5\% hydrochloric acid (Scheme 14). Thiochromanones can alternatively be synthesized from the isolated nitroderivatives $\mathbf{4 5}$ by the Nef reaction.

\subsubsection{Homo- and hetero-cycles from $\beta$-nitrothiophenes. A dichotomic thermal vs. base- induced cyclization}

The same $i$ ) to $v$ ) step sequence which leads from 41 to 44 in Scheme $14(\mathrm{R}=\mathrm{H})$, has been successfully applied (Scheme 15) to the most simple $\beta$-nitrothiophene, i.e. 3-nitrothiophene itself (10a), to prepare the nitrobutadienic intermediates 47.

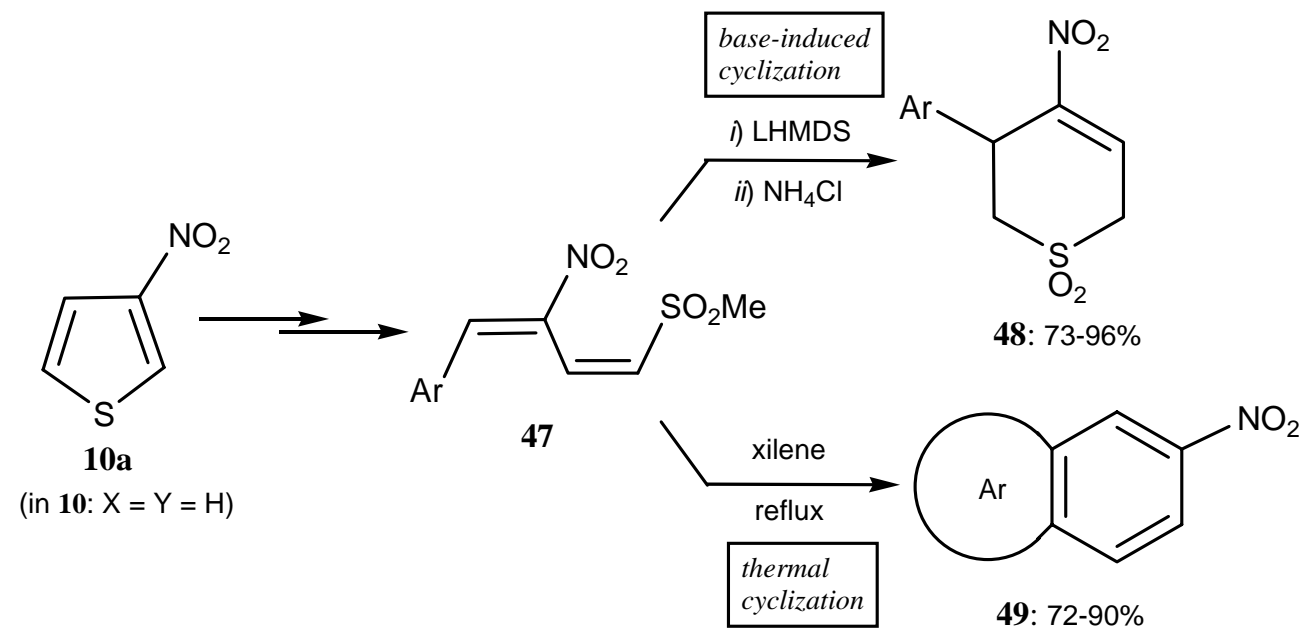

Scheme 15. Thermal and base-induced cyclization of intermediates from the ring-opening of 3nitrothiophene. 
Depending on the reaction conditions, 47 has actually revealed to undergo an interesting dichotomic intramolecular cyclization process. Treatment with LHMDS in experimental conditions similar to those employed on the sulfones from 3-nitrobenzo[b]thiophene, leads to thiopyran S,S-dioxides (48), while heating in refluxing xylene leads to benzocondensed derivatives. This thermal cyclization leads to the benzocondensed derivatives (49) in more than satisfactory yields and thus represents an appealing synthetic route to compounds which are generally not easy to prepare via more conventional methods. The mechanism of this reaction involves a disrotatory thermal $6 \pi$-electrocyclization driven forward by a very efficient syn $\beta$ elimination of methanesulfinic acid. The efficiency of the final aromatization step is conceivably responsible for the reaction conditions, which are exceptionally mild with respect to electrocyclic processes where electrons of aromatic systems are involved. ${ }^{7 a}$

As far as the base-catalyzed process is concerned, the neat result is again an enlargement of the starting thiophene ring with the formation of thiopyran $S, S$-dioxides $\mathbf{4 8}$ via deprotonation and Michael-type cyclization onto the nitrovinyl moiety, followed by selective protonation of the carbon adjacent to the sulfonyl group. Thus, at variance with the benzothiophene system described above, the isolated thiopyrans $\mathbf{4 8}$ still contain a nitrovinyl moiety. This outcome simplifies the stereochemical aspects and only one racemic mixture is isolated throughout. The nitroethylenic moiety may be successively subjected to further tranformations to obtain the corresponding oximes and carbonyl derivatives: the latter, difficult to isolate, where characterized as the relevant dinitrophenylhydrazones.

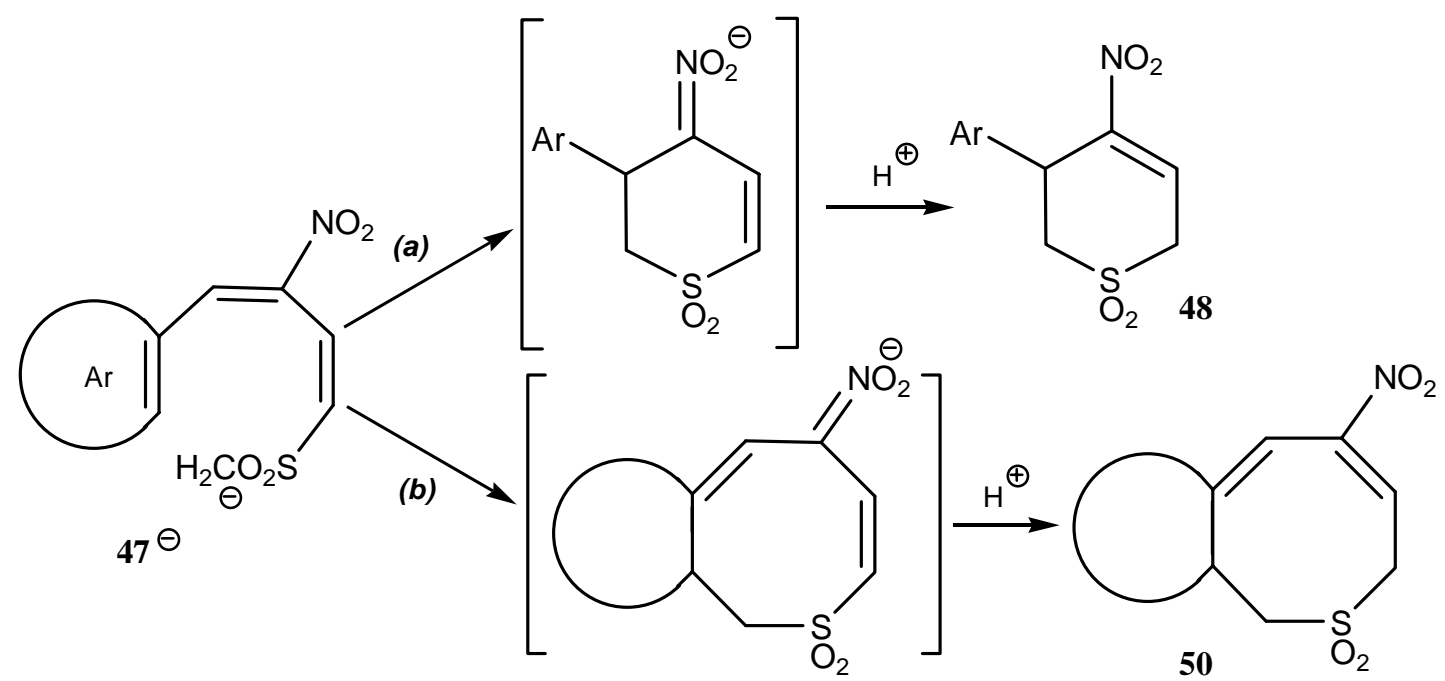

Scheme 16. Competitive Michael-type cyclizations onto nitrovinyl vs. aryl.

But another surprising reactivity shows up when the aryl in $\mathbf{4 7}$ is a naphthyl or a thienyl group. Actually (Scheme $16 ; \mathbf{4 7}^{\ominus}$ has been drawn in the s-trans-configuration for an easier visualization of the intramolecular process) in these case the base-induced cyclization onto the nitrovinyl moiety (route $a$ ) competes with cyclization onto the aryl moiety itself (route $b$ ), 
leading to the interesting condensed derivatives $\mathbf{5 0}$ characterized by unusual 8-membered heterorings. Their formation is at present under examination, but the competitivity of this second pathway is surely temperature dependent and becomes substantial at $-78{ }^{\circ} \mathrm{C}$. ${ }^{22}$

For comparison sake, it is worthwhile to remember (Scheme 17) the behaviour shown by the 1-aryl-2-nitro-3-phenylsulfonyl butadienes 51, obtained when applying the same sequence of reactions (steps $i-v$, Scheme 14) to the 3-phenylsulphonyl-4-nitrothiophene (10b). Much as butadienes 47 (Scheme 15), compounds 51 undergo thermal cyclization smoothly, leading to the corresponding benzocondensed derivatives 52 in high yields; ${ }^{7 \mathrm{c}}$ on the contrary, when we tried to apply the base-induced process, just described for the 44 to 45 (Scheme 14) and the 47 to 48 (Scheme 15 and 16) transformations, we could obtain no significant evidence for the formation of the corresponding cyclized products.

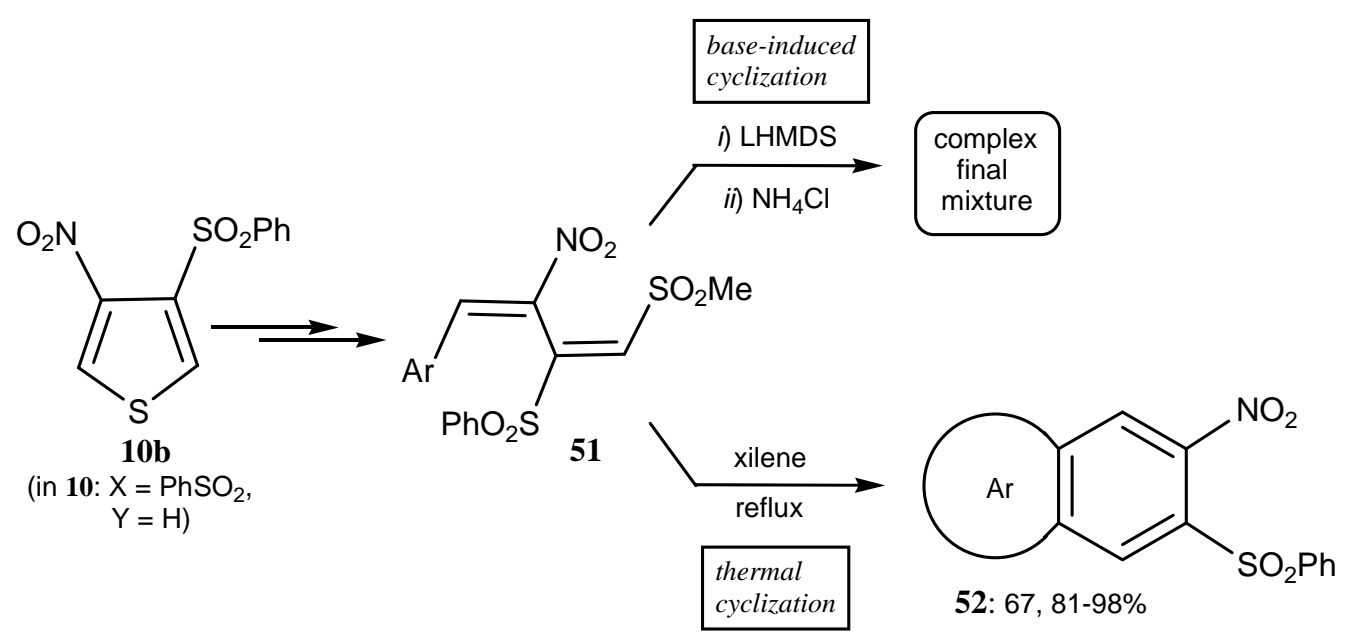

Scheme 17. Thermal cyclization of intermediates from the ring-opening of 3-phenylsulfonyl-4nitrothiophene.

\subsubsection{Isoxazoles from 2-methylthio-4-nitrothiophene}

Among the butadienic building-blocks obtainable by the ring-opening reaction of $\beta$-NT's, we found of particular interest (Scheme 18) the (E)-1,2-bis(methylthio)-3-nitro-4-pyrrolidino-1,3butadiene 53, that is the ring-opening product of 2-methylthio-4-nitrothiophene (10c). Actually, the replacement of the pyrrolidino group with the aryl of an aryl Grignard reagent leads to intermediates 54, whose nitrovinyl moiety can be then reduced to give benzyl vinyl oximes 55; the treatment in acetonitrile at reflux with an acidic ion-exchange resin (amberlyst $15^{\circledR}$ ) of these compounds, which possess two geminal methylthio substituents, finally allows the cyclization to the 3-arylmethyl-5-(methylthio)isoxazoles $56 .^{7 \mathrm{~d}}$ 


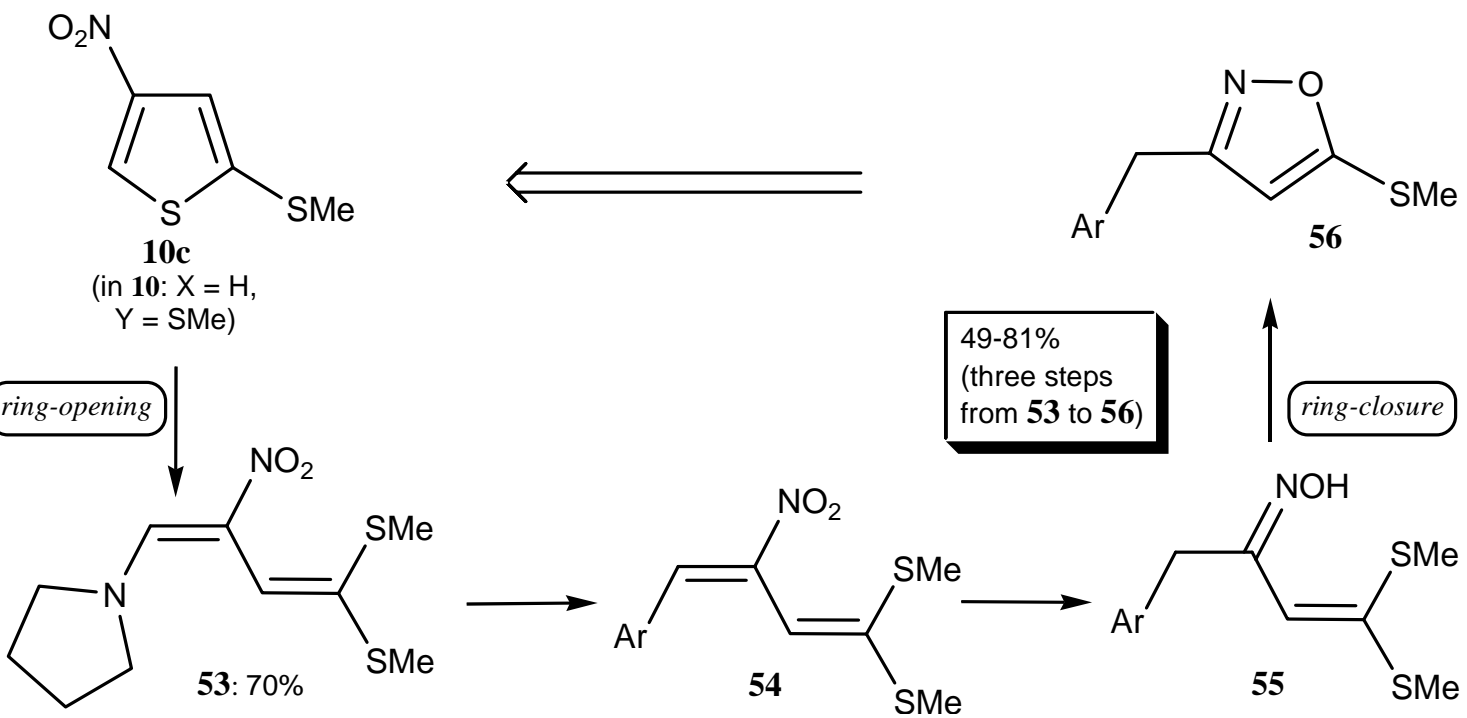

Scheme 18. 3-Arylmethyl-5-(methylthio)isoxazoles from 2-methylthio-4-nitrothiophene.

\section{Acknowledgements}

Financial support was provided by grants from the Universities of Genova and of Bologna, and from Ministero dell’Istruzione, dell’Università e della Ricerca (PRIN 2004).

\section{References and Notes}

1. Dell’Erba, C.; Spinelli, D.; Leandri, G. Gazz. Chim. Ital. 1969, 99, 535.

2. Dell’Erba, C.; Spinelli, D.; Leandri, G. J. Chem. Soc., Chem. Commun. 1969, 549.

3. Bianchi, L.; Dell'Erba, C.; Maccagno, M.; Mugnoli, A.; Novi, M.; Petrillo, G.; Tavani, C. Eur. J. Org. Chem. 2004, 3566.

4. For reviews see: (a) Spinelli, D.; Consiglio, G.; Dell'Erba, C.; Novi, M. in The Chemistry of Heterocyclic Compounds, Thiophene ad its Derivatives Gronowitz, S., Ed., J. Wiley, New York, 1991, Vol. 44, pp 295-396. (b) Dell'Erba, C.; Novi, M.; Petrillo, G.; Tavani, C. In Topics in Heterocyclic Systems: Synthesis, Reactions and Properties Attanasi, O. A.; Spinelli, D., Eds., Research Signpost: Trivandrum, India, 1996, Vol. 1, pp 1-12. (c) Consiglio, G.; Spinelli, D.; Dell'Erba, C.; Novi, M.; Petrillo, G. Gazz. Chim. Ital. 1997, 127, 753.

5. Guanti, G.; Dell’Erba, C.; Leandri, G.; Thea, S. J. Chem. Soc., Perkin Trans. 1 1974, 2357.

6. Dell'Erba, C.; Gabellini, A.; Novi, M.; Petrillo, G.; Tavani, C.; Cosimelli, B.; Spinelli, D. Tetrahedron 2001, 57, 8159.

7. (a) Bianchi, L.; Dell'Erba, C.; Maccagno, M.; Petrillo, G.; Rizzato, E.; Sancassan, F.; Severi, E.; Tavani, C. J. Org. Chem. 2005, 70, 8734. (b) Bianchi, L.; Dell'Erba, C.; Maccagno, M.; 
Morganti, S.; Novi, M.; Petrillo, G.; Rizzato, E.; Sancassan, F.; Severi, E.; Spinelli, D.; Tavani, C. Tetrahedron 2004, 60, 4967. (c) Bianchi, L.; Dell'Erba, C.; Maccagno, M.; Mugnoli, A.; Novi, M.; Petrillo, G.; Sancassan, F.; Tavani, C. J. Org. Chem. 2003, 68, 5254. (d) Bianchi, L.; Dell'Erba, C.; Gabellini, A.; Novi, M.; Petrillo, G.; Tavani, C. Tetrahedron 2002, 58, 3379.

8. (a) Surange, S. S.; Kumaran, G.; Rajappa, S.; Rajalakshmi, K.; Pattabhi, V. Tetrahedron 1997, 53, 8531. (b) Surange, S. S.; Rajappa, S. Tetrahedron Lett. 1998, 39, 7169.

9. Petrillo, G. et al., unpublished results.

10. Dell'Erba, C.; Mele, A.; Novi, M.; Petrillo, G.; Stagnaro, P. Tetrahedron 1992, 48, 4407.

11. Dell'Erba, C.; Mugnoli, A.; Novi, M.; Pertici, M.; Petrillo, G.; Tavani, C. Eur. J. Org. Chem. 1999, 431.

12. Dell'Erba, C.; Gabellini, A.; Mugnoli, A.; Novi, M.; Petrillo, G.; Tavani, C. Tetrahedron 2001, 57, 9025.

13. Dell'Erba, C.; Novi, M.; Petrillo, G.; Spinelli, D.; Tavani, C. Tetrahedron 1996, 52, 3313.

14. Dell'Erba, C.; Novi, M.; Petrillo, G.; Stagnaro, P. J. Heterocyclic Chem. 1994, 31, 861.

15. Armani, V.; Dell'Erba, C.; Novi, M.; Petrillo, G.; Tavani, C. Tetrahedron 1997, 53, 1751.

16. Bianchi, L.; Dell’Erba, C.; Gasparrini, F.; Novi, M.; Petrillo, G.; Sancassan, F.; Tavani, C. Arkivoc 2002, 142. Armaroli, T.; Dell'Erba, C.; Gabellini, A.; Gasparrini, F.; Mugnoli, A.; Novi, M.; Petrillo, G.; Tavani, C. Eur. J. Org. Chem. 2002, 1284.

17. Petrillo, G. et al., unpublished results.

18. Petrillo, G. et al., unpublished results.

19. Dell'Erba, C.; Mugnoli, A.; Novi, M.; Pani, M.; Petrillo, G.; Tavani, C. Eur. J. Org. Chem. 2000, 903.

20. Dell'Erba, C.; Giglio, A.; Mugnoli, A.; Novi, M.; Petrillo, G.; Stagnaro, P. Tetrahedron 1995, 51, 5181.

21. Petrillo, G. et al., unpublished results.

22. Petrillo, G. et al., unpublished results. 